\title{
Study of Eppler 423 Airfoil with Gurney Flap and Vortex Generators
}

\author{
Sushil Chandra, Rajan Tyagi* \\ Aerospace Engineering Department, IIT Bombay, Mumbai, India \\ Email: maahe_sf@yahoo.com, ^rajantyagi85@gmail.com
}

How to cite this paper: Chandra, S. and Tyagi, R. (2020) Study of Eppler 423 Airfoil with Gurney Flap and Vortex Generators. Advances in Aerospace Science and Technology, 5, 1-19.

https://doi.org/10.4236/aast.2020.51001

Received: November 11, 2019

Accepted: January 3, 2020

Published: January 6, 2020

Copyright $\odot 2020$ by author(s) and Scientific Research Publishing Inc. This work is licensed under the Creative Commons Attribution International License (CC BY 4.0).

http://creativecommons.org/licenses/by/4.0/

(c) (i) Open Access

\begin{abstract}
In the past extensive research has been carried out, to study the effect of Gurney flap (GF) on symmetric and cambered airfoil for its usage in low Reynolds number regime. Use of GF at the trailing edge of the airfoil enhances the lift due to increase in the effective camber of the airfoil, which in turn improves the aerodynamic efficiency i.e. $C_{l} / C_{d}$. In the present study, Eppler 423 airfoil is used to first understand the aerodynamics of such a highly cambered airfoil and later GF of various sizes were added on it to understand the change in flow dynamics achieved by adding the GF and their impact on aerodynamic parameters such as $C_{b} C_{d}$ and $C_{l} / C_{d}$. Eppler 423 being a highly cambered airfoil produces high lift coefficient and smoother stall and by adding the GF of various sizes the performance of Eppler 423 improves tremendously and reason for this enhanced performance and effect of size of GF are presented in this paper. Vortex Generators (VG) generate counter rotating vortices that allow the flow to remain attached even at high angles of attack. Also, effect of adding VG at the leading edge of Eppler 423 aerofoil is presented in this paper. At last, results obtained from combination of VG at leading edge and GF at trailing edge on Eppler 423 aerofoil are discussed at length.
\end{abstract}

\section{Keywords}

Eppler 423 Airfoil, Gurney Flap, Vortex Generators, Aerodynamic Efficiency, Stall

\section{Introduction}

The Gurney flap is a small flap (like tab in aircraft wing trailing edge), added at the trailing edge of an airfoil or wing at right angle to the pressure surface. Generally its height varies between $1 \%-2 \%$ of chord or inside boundary layer [1]. Addition of Gurney Flap to enhance lift of an airfoil/wing is not a new concept 
to be thought of; it was erstwhile originated from racing cars, but its roots can be traced back to 1935 by E. F. Zaparka [1] patent US Patent Re19412. Its origin and usage on race car was introduced by Late Dan Gurney (1931-2018) a racing car driver, who later owned a racing car company AAR (All American Racers). He improvised the car in 1971, which was underperforming, to the winning car (as a manager post-retirement from racing), this invention was inspired from spoilers attached to the rear of bodywork to cancel lift by certain teams in the 1950s [1]. Gurney was able to use the device in racing for several years before its real purpose became known. Later, he discussed his ideas with an aerodynamicist and wing designer Bob Liebeck [2] of Douglas Aircraft Company. Liebeck tested the device, which he then named the "Gurney flap" and confirmed Gurney's field test results using a $1.25 \%$ chord flap on a Newman symmetric airfoil. An experimental study was conducted by Bob Liebeck [2] on a Newman aerofoil. He found that the GF with only $1.25 \%$ chord length gave high-lift coefficient by increasing lift but also reducing drag at the same time. Liebeck also found that the flap height should be between $1 \% \mathrm{C}$ and $2 \% \mathrm{C}$ to maximize the aerodynamic benefits from this simple high-lift device. He also concluded that flaps with a height of more than $\mathrm{h}=2 \% \mathrm{C}$ would significantly increase the drag. Liebeck from his results proposed the formation of two counter-rotating vortices downstream of the Gurney Flap (Figure 1). It is evident that both lift and drag coefficients increase with an increase in the height of the Gurney flap. Giguere et al. [3] conducted the study to understand the effects of the mounting location of the Gurney flap on airfoil. They found that the increment of lift coefficient had decreased when the GF has shifted forward away from the trailing edge, weakening the lift-enhancing effects of the flap. Also Giguere et al. [3] suggested that the size of the optimum GF for the best lift-to-drag ratio is determined by the flow condition at the trailing edge at the pressure side of the airfoil. It was recommended that the flap should be submerged in the boundary layer, which was also confirmed by Li [4] through experiments. Li et al. [5] studied the effect of mounting angle of GF on aerodynamic performance of NACA0012 airfoil and found that with increase in mounting angle $C_{l}$ increases as compared to clean

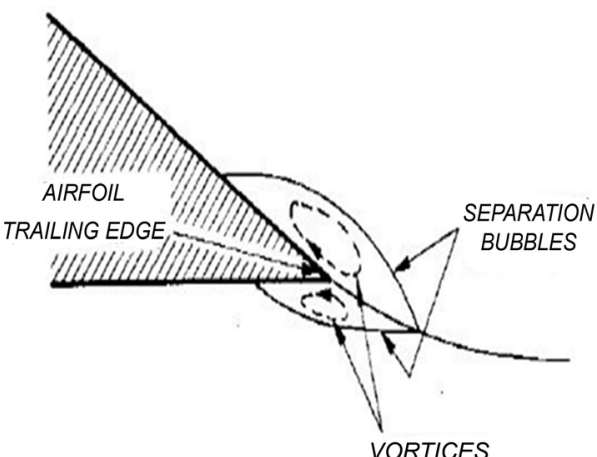

(a)

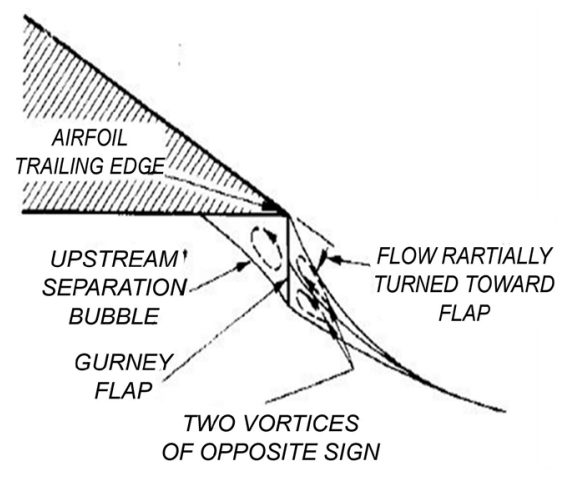

(b)

Figure 1. Flow over aerofoil with and without Gurney flap [2]. (a) Conventional aerofoil at moderate $C_{\dot{p}}(\mathrm{~b})$ Hypothesized flow near Gurney flap. 
aerofoil. Neuhart et al. [6] investigated the effects of saw-toothed GF on airfoil aerodynamics, and found that saw-toothed flaps can make the flow around the trailing edge more three-dimensional. Similarly, investigations by Vijgen et al. [7], indicated that the saw-toothed GF could increase the lift and reduce the drag, thereby greatly improving the lift-to-drag ratio as compared to a plain GF of the same height. Mitchell et al. [8] in their investigation indicated that with increase in thickness of GF, $C_{l}$ is seen to decrease also zero-lift angle of attack shift is less pronounced with increasing thickness, suggesting that thicker flaps reduce the effective camber. Roy et al. [9] and Yachen Li et al. [10] found in their experiments on NACA 0011 aerofoil that addition of the Gurney flap adds camber to the aerofoil and thus increases the coefficient of lift and effect of GF on symmetrical aerofoil is much more when compared with cambered aerofoil. Also, P. Giguère et al. [11] found that addition of GF on low speed aerofoils tends to increase the effective camber of the aerofoil and thus increases the lift coefficient. As evident from the existing literature both GF and VG when added independently have a positive effect on the performance of Eppler 423 aerofoil as both increases the aerodynamic efficiency of the Eppler 423 aerofoil. In the present study, firstly behavior of clean Eppler 423 aerofoil is studied than a comparison is made with configuration having GF and VG only followed by configuration having both GF and VG.

\section{Materials and Methods}

A low speed suction type, open circuit wind tunnel was used for the conduct of all experiments during this study as shown in Figure 2. The wind tunnel has a test section cross-section dimension of $920 \mathrm{~mm} \times 920 \mathrm{~mm}$. Both sides of the test section have a Perspex window for easy viewing. The inlet of the wind tunnel has a bell mouth for ensuring smooth inlet of air into the wind tunnel. The inlet air then passes through the honeycomb straighteners and a series of four wire screens with varying mesh sizes. Subsequently, before the air enters the test section, the flow is allowed to settle in the settling chamber. The airfoil used for this study was Eppler 423, the airfoil details are highlighted in Table 1. The airfoil is made from wood; the model had a total of 28 pressure ports on the suction side, 26 pressure ports on the pressure side and one pressure port at the leading edge. Copper tubes of $0.5 \mathrm{~mm}$ were inserted under the pressure ports and across the span in the grooves made in the airfoil. Airfoil was smoothened using adhesive putty. Eppler 423 airfoil was fixed inside the wind tunnel in an inverted position with a straight plywood sheet, and groove on another side. All the pressure taps were protruding outside the slot and then to pressure scanner. All readings in the experiments were taken by keeping the flow velocity to $20 \mathrm{~m} / \mathrm{s}$. A pitot-static tube connected to digital manometer was used to measure the dynamic pressure, from which the velocity is calculated. All the pressure tapings from the airfoil were attached to multi-channel pressure selector panel, the output of which was connected to a digital manometer. The readings from manometer gave pressure 


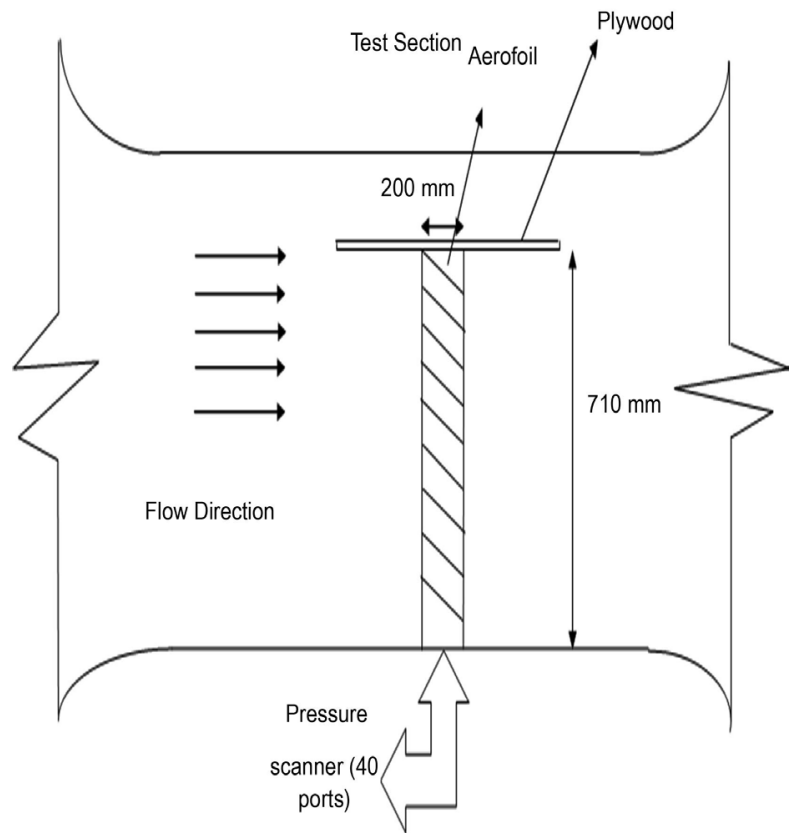

(a)

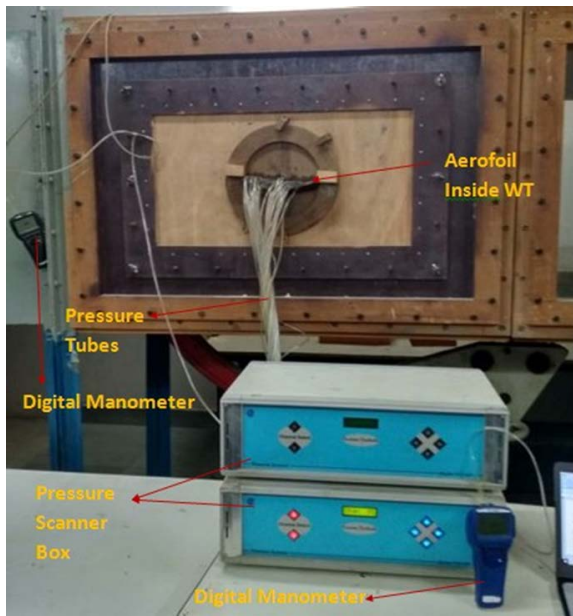

(b)

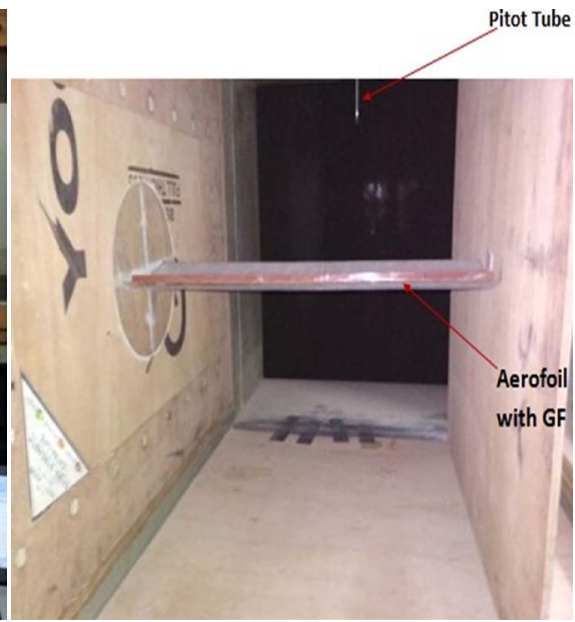

(c)

Figure 2. Aerofoil setup. (a) Wind tunnel schematic; (b) Setup; (c) Eppler with GF.

Table 1. Dimensions of Eppler 423 Aerofoil.

\begin{tabular}{|c|c|c|c|c|}
\hline Configuration & Max Thickness & Max Camber & Span (mm) & Chord (mm) \\
\hline Eppler 423 & $12.5 \%$ at $23.7 \%$ chord & $9.5 \%$ at $41.4 \%$ chord & 760 & 200 \\
\hline
\end{tabular}

difference between the selected channel (connected to a port on airfoil) and static pressure from the Pitot-static tube. The desired channel can be selected for measurement, and the pressure reading at the manometer is noted. Each channel of the multi-channel selector panel was numbered and marked to a pressure port on the airfoil.

Gurney flap was used on the trailing edge with different configurations; 3D printing was utilized for fabrication of Gurney flaps as shown in Figure 3(a). 


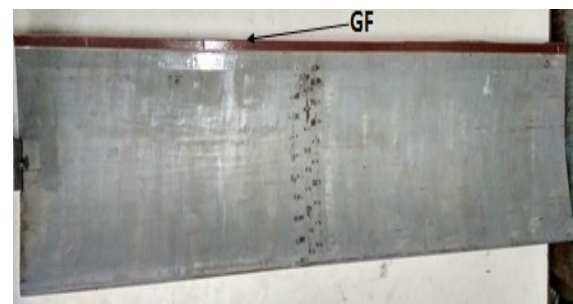

(a)

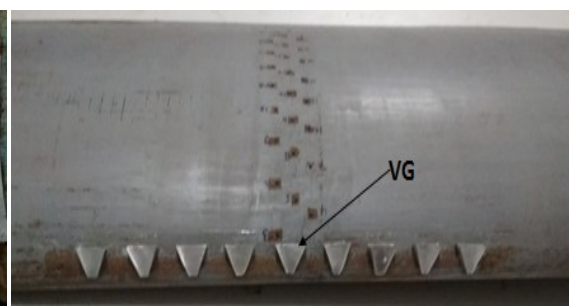

(b)

Figure 3. Aerofoil geometry with GF and VG. (a) Eppler with GF; (b) Eppler with VG.

Various configurations utilized were in \% of chords, namely $2 \%, 3 \%, 4 \%$ and $5 \%$ of chord $(200 \mathrm{~mm})$. Fabricated models were in L shape with $5 \mathrm{~mm}$ base, and other dimensions are in $\%$ of chord.

VG was used like in conventional aircraft, in which the VG's are attached on the leading edge as shown in Figure 3(b). The patterns were chosen such that the counter-rotating vortices were formed, which will prevent flow separation and allow flow to remain attached, which is evident from the modified pressure distribution. The height, as well as dimensions facing the flow of VG, is $2.5 \%$ of chord as shown in Figure 4 and Figure 5. Total of nine VG's were used on the airfoil leading edge, equally spaced at $2 \%$ of chord. Different configurations tested are mentioned in Table 2.

\section{Results and Discussion}

\subsection{Variation of Coefficient of Pressure (Cp) with and without GF}

Figure 6(a) shows the distribution of pressure around a clean airfoil on the upper and lower surface against $x / c$. The pressure distribution modifies as the AoA is increased, the flow separation occurs on the suction surface at 10 degrees, the peak of the suction surface goes up to -4 at 14 degrees AoA. The area keeps on increasing as the AoA is increased beyond -4 , as a result, lift as well as pressure drag also increases, at around 10 degree AoA stall occurs which results in reduction of lift.

Figure $6(\mathrm{~b})$ shows the modified pressure distribution over the airfoil at $2 \%$ Gurney flap. It is observed that addition of the Gurney flap alters the pressure distribution over the pressure surface that results in the enhanced lift, also addition of Gurney flap results in increase in effective camber of the airfoil. However, the effect is more prominent on symmetric airfoil than on cambered airfoils. As, Eppler 423 is a highly cambered airfoil and addition of gurney flap results in further increase in camber that results in an additional increment in lift compared to clean airfoil, but there is a penalty in terms of reduction in stall angle.

Figures 7(a)-(c) show the modified pressure distribution over Eppler 423 airfoil with 3\%, 4\% and 5\% GF. It was observed that with increase in GF height the separation tends to occur early when compared with clean wing and airfoil with $2 \%$ GF. The reason for this is that with increase in GF height the effective camber of the airfoil increases which leads to early flow separation. 


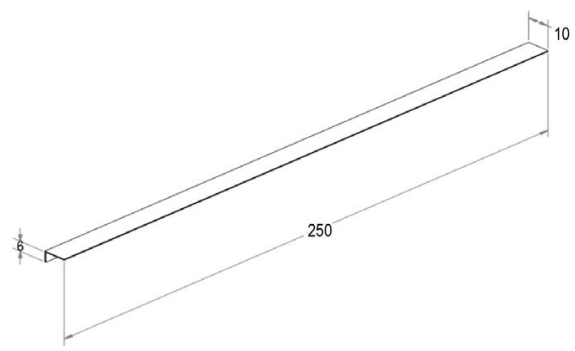

(a)

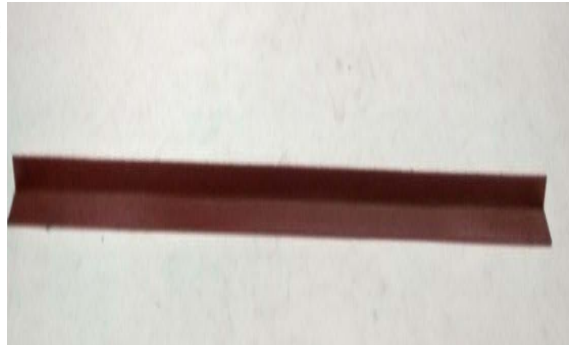

(b)

Figure 4. Gurney flap. (a) Gurney flap dimensions; (b) Gurney flap used in experiments.

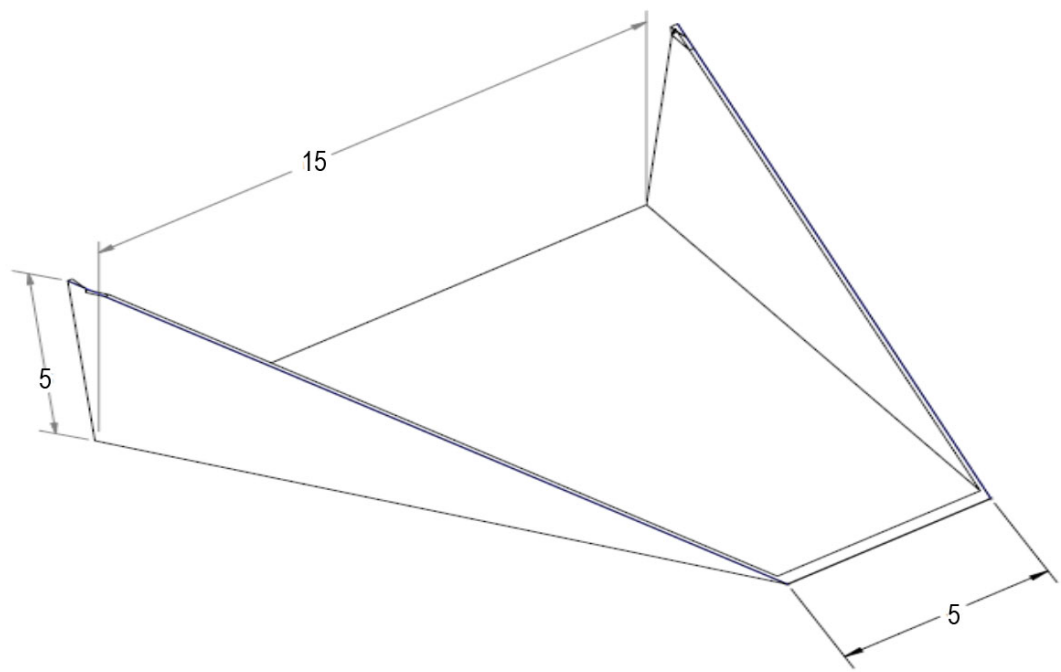

Figure 5. Vortex generator.

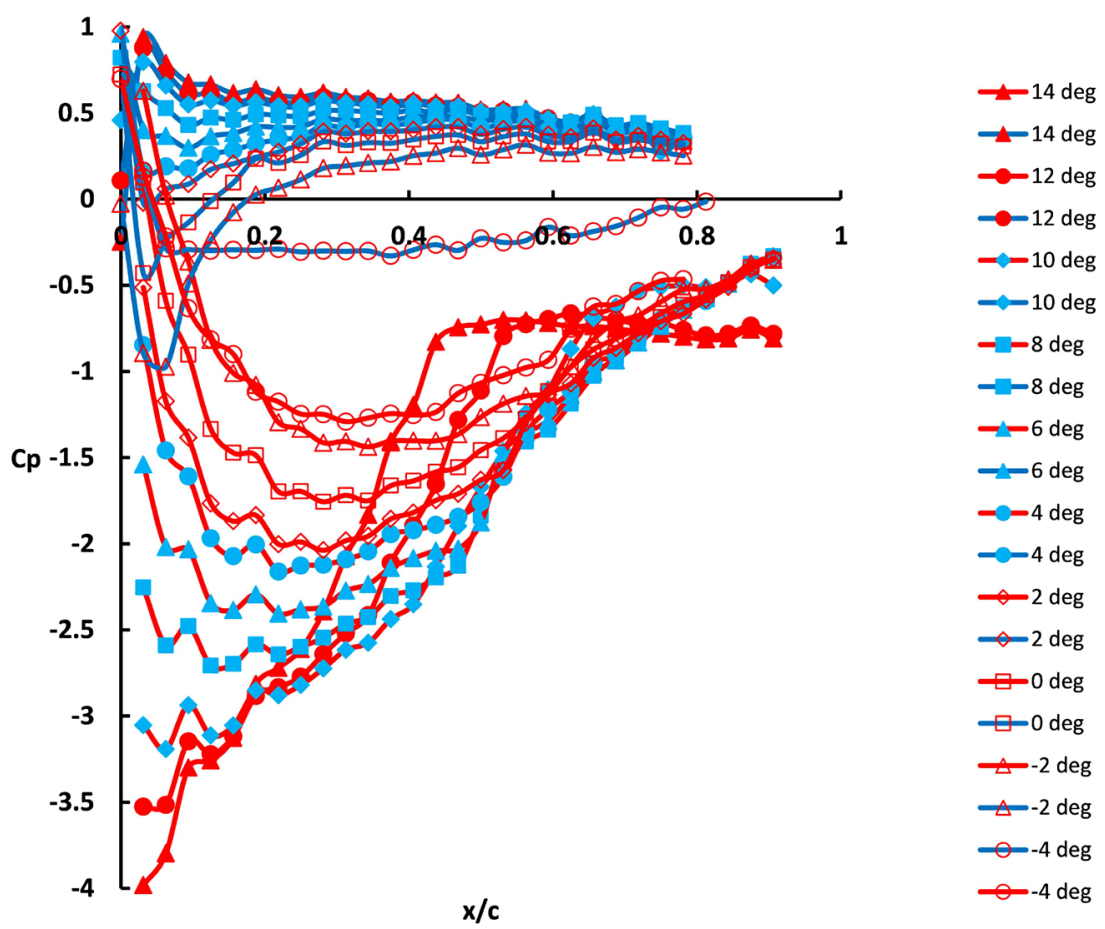

(a) 

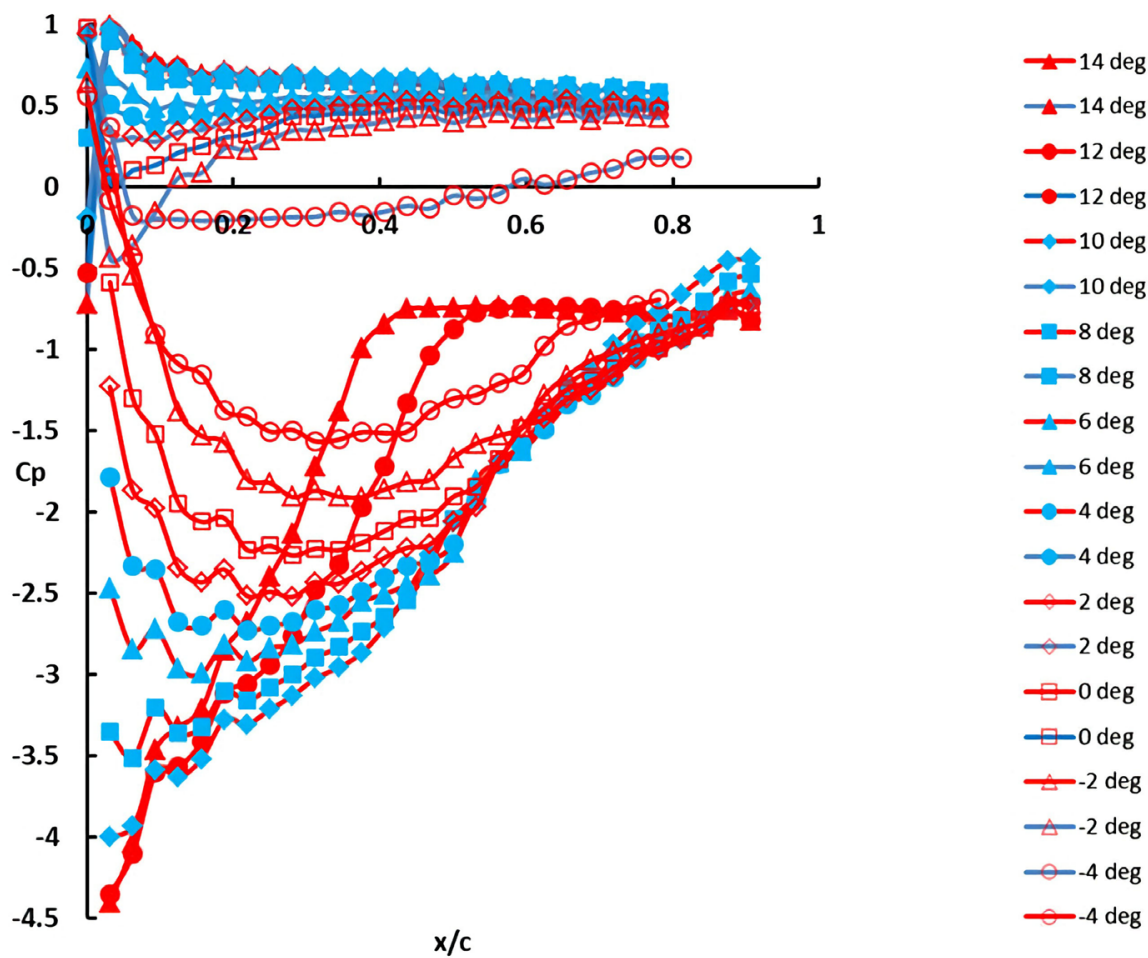

(b)

Figure 6. Pressure distribution on clean wing and with GF. (a) Pressure distribution on clean wing; (b) Pressure distribution with 2\% GF.

Table 2. Configurations tested in wind tunnel.

\begin{tabular}{cc}
\hline Configuration & AoA (in Degrees) \\
\hline Airfoil with 2\% GF & $-4,-2,0,2,4,6,8,10,12$ and 14 \\
Airfoil with 3\% GF & $-4,-2,0,2,4,6,8,10,12$ and 14 \\
Airfoil with 4\% GF & $-4,-2,0,2,4,6,8,10,12$ and 14 \\
Airfoil with 5\% GF & $-4,-2,0,2,4,6,8,10,12$ and 14 \\
Airfoil with 2\% GF and VG & $-4,-2,0,2,4,6,8,10,12$ and 14 \\
Airfoil with 3\% GF and VG & $-4,-2,0,2,4,6,8,10,12$ and 14 \\
Airfoil with 4\% GF and VG & $-4,-2,0,2,4,6,8,10,12$ and 14 \\
Airfoil with 5\% GF and VG & $-4,-2,0,2,4,6,8,10,12$ and 14 \\
\hline
\end{tabular}

\subsection{Variation of Coefficient of Pressure (Cp) with Gurney Flap (GF) and Vortex Generators (VG)}

The effect of addition of GF is that at the lower surface the flow remains attached while at the same time the flow separates from the upper surface at higher angles of attack. This separation can be reduced by the addition of VG at the leading edge (conventionally). Figure 8 shows pressure distribution over Eppler 423 with VG only, and it can be seen that even at higher angles of attack, there is no flow separation from the upper surface. Addition of GF, with VG on the leading-edge enhances the lift as can be seen from the Figure 9 and Figure 10, there 
is a marked improvement in the performance of the highly cambered Eppler 423 airfoil as compared to the airfoil with only GF. It can be seen that the stall angle has been increased thus delaying the stall.

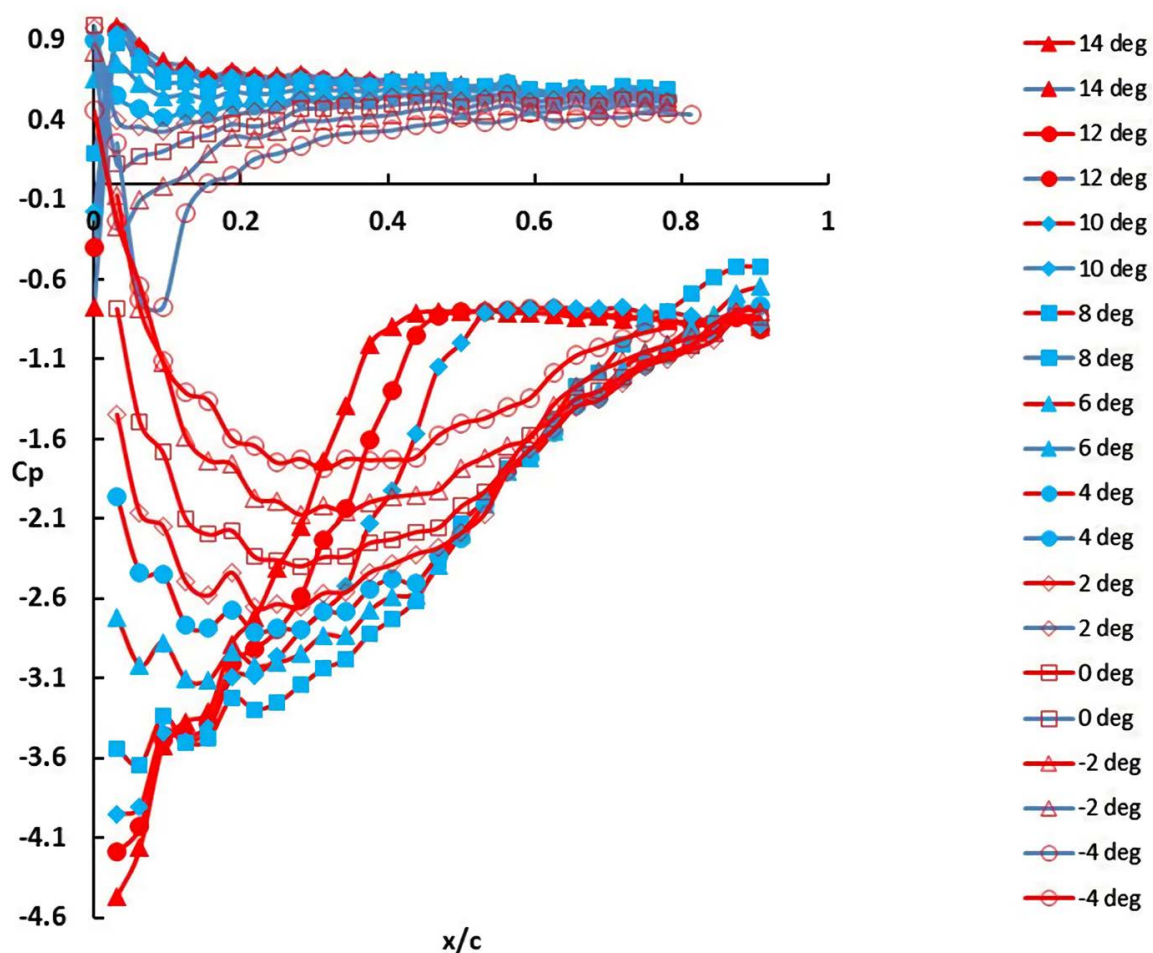

(a)

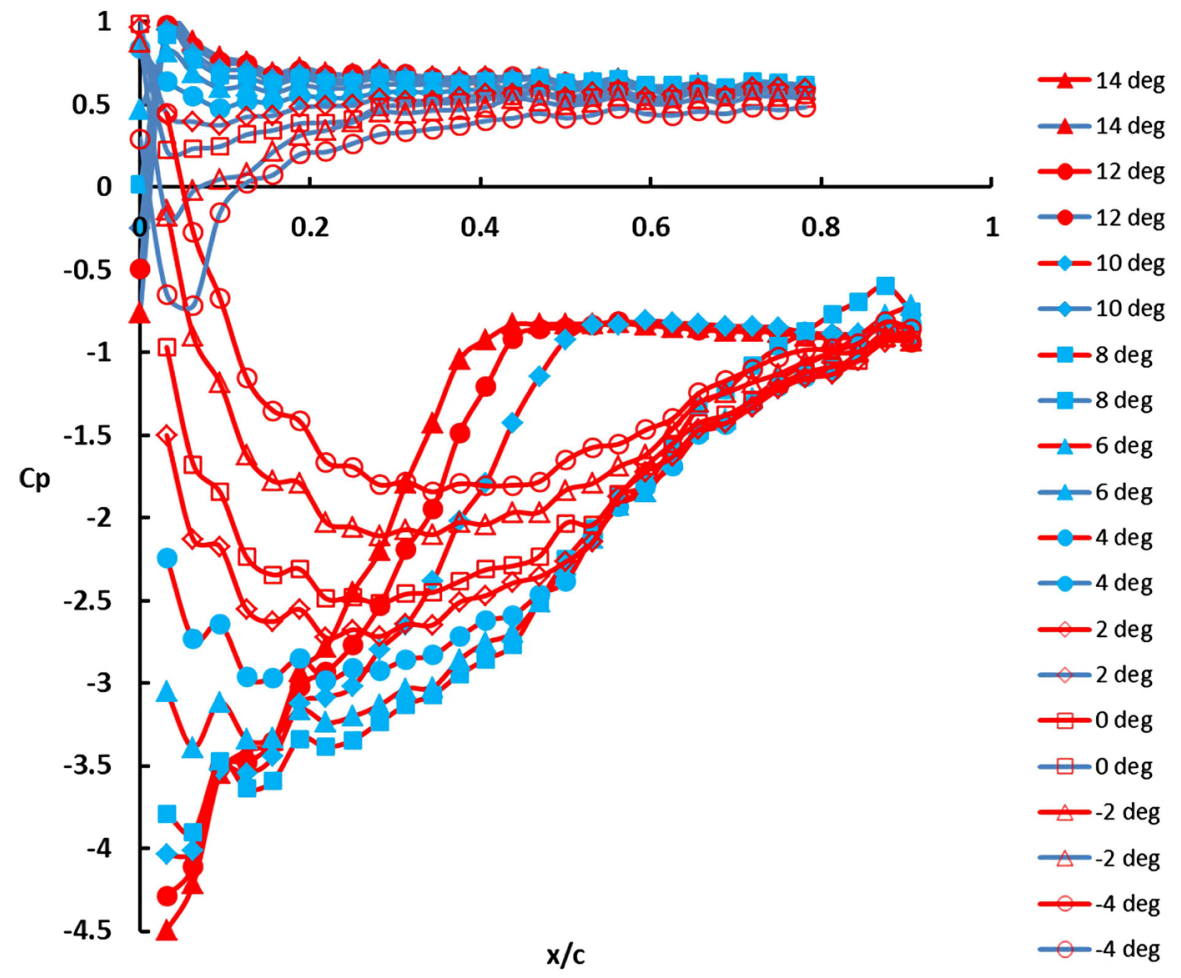

(b) 


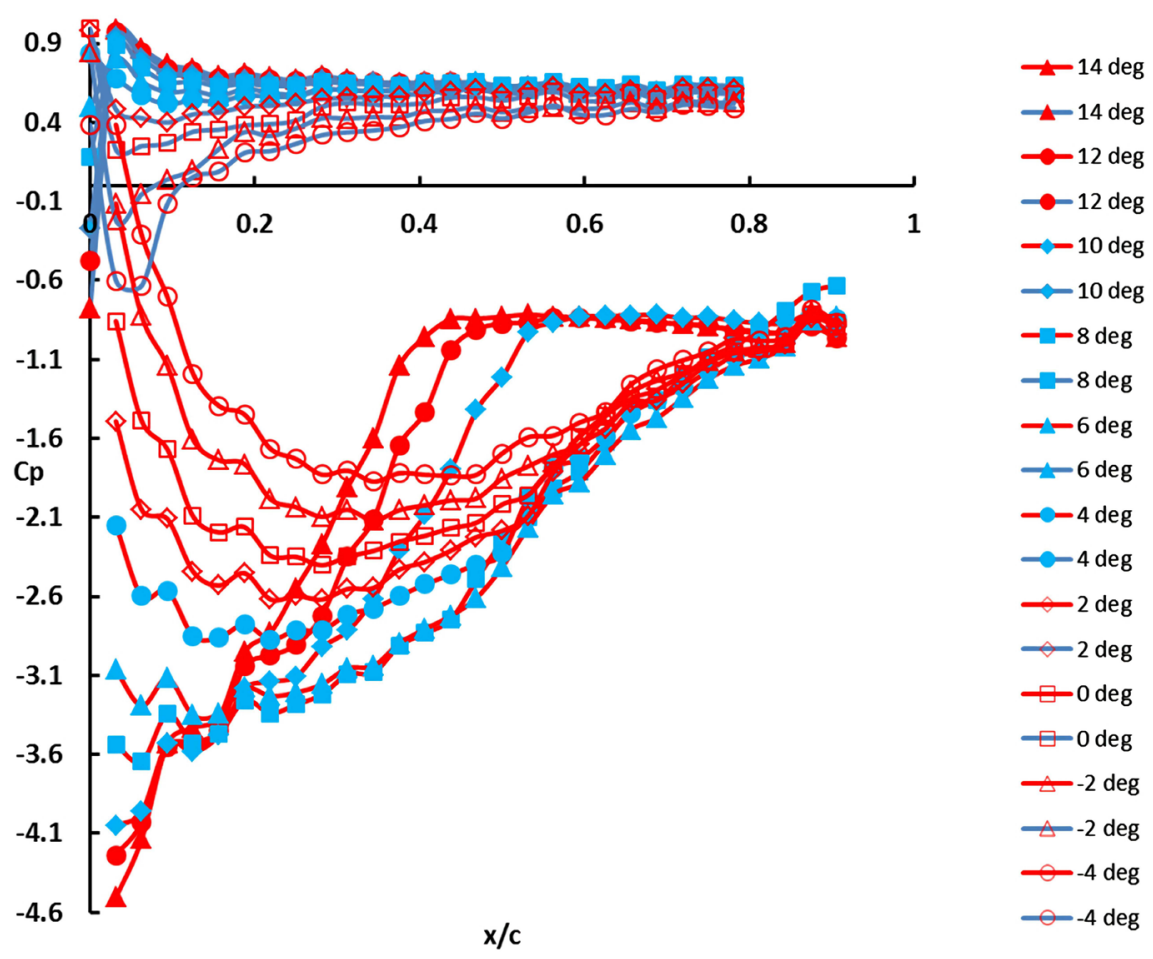

(c)

Figure 7. Pressure distribution with GF. (a) Pressure distribution with 3\% GF; (b) Pressure distribution with 4\% GF; (c) Pressure distribution with 5\% GF.

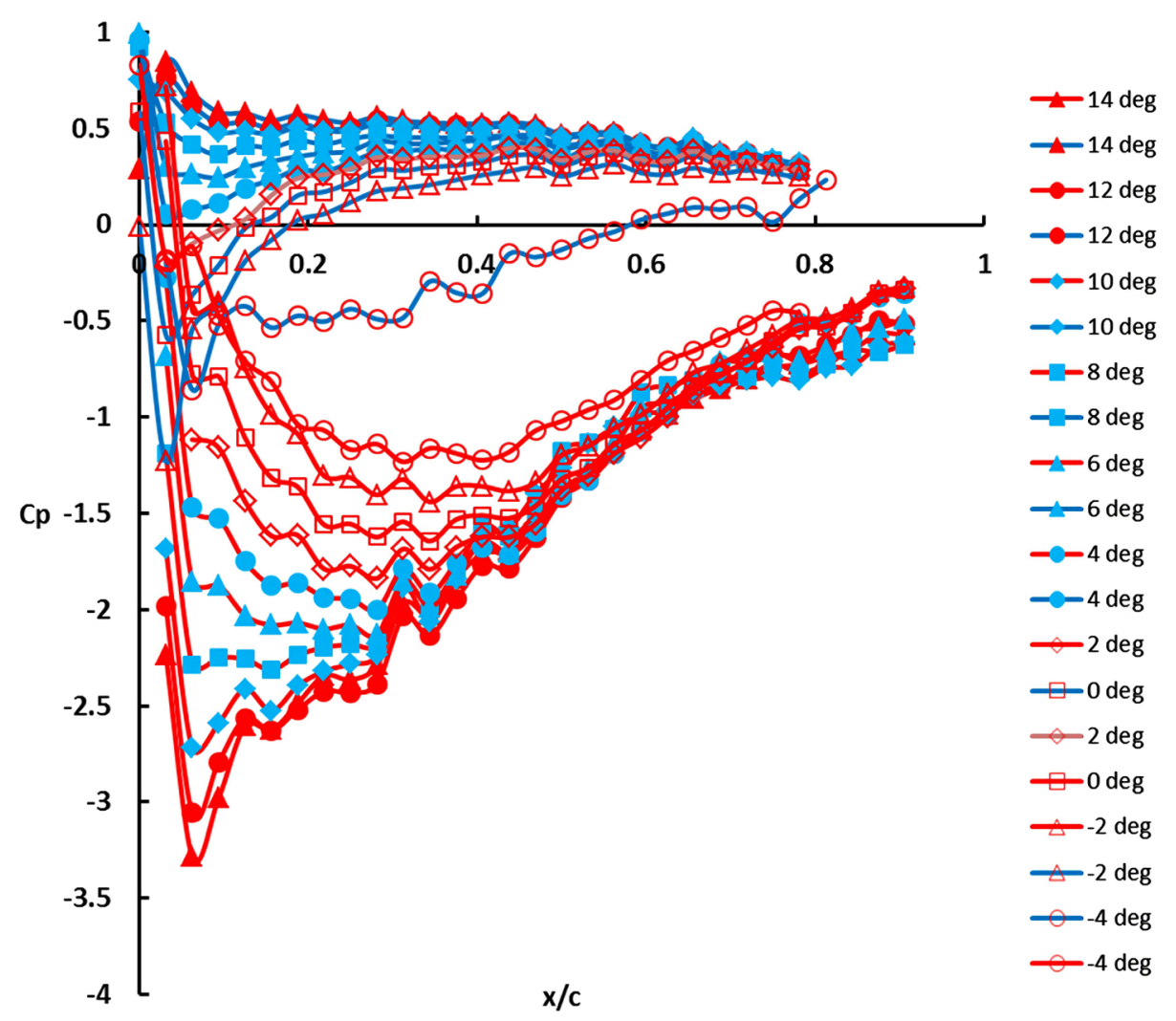

Figure 8. Pressure distribution with VG. 

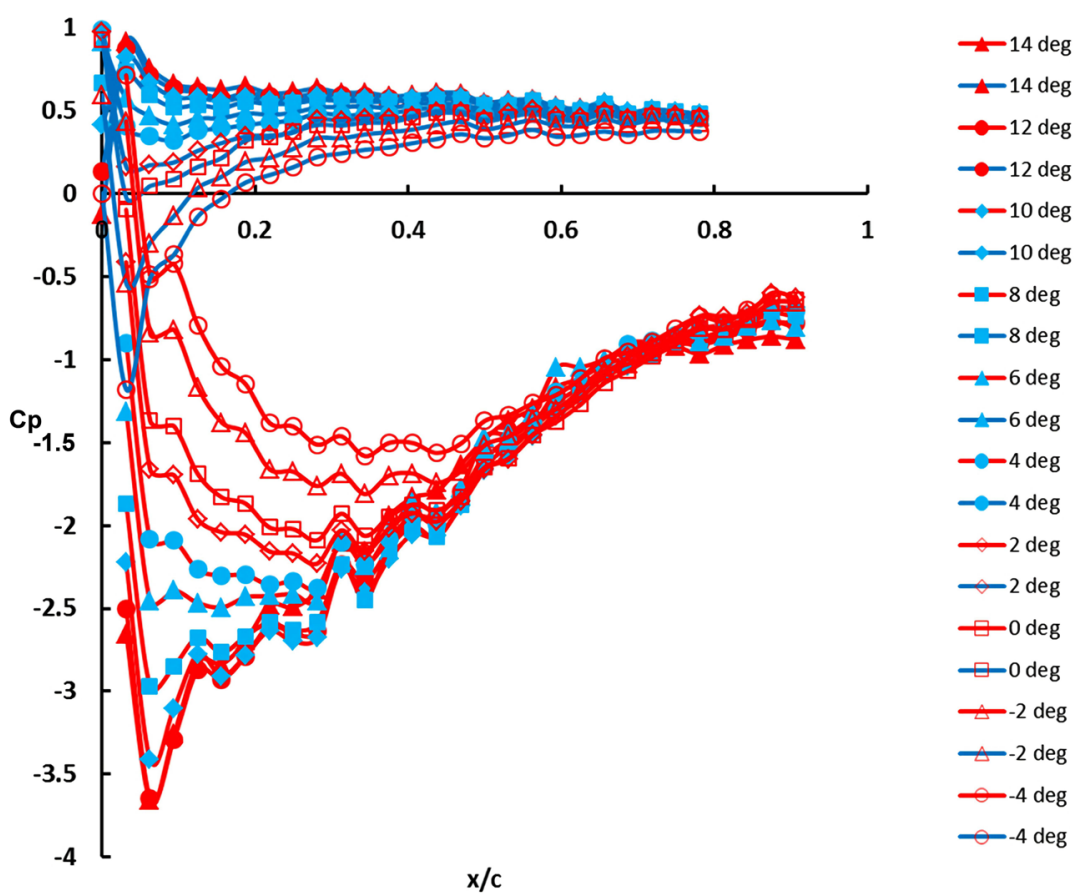

(a)
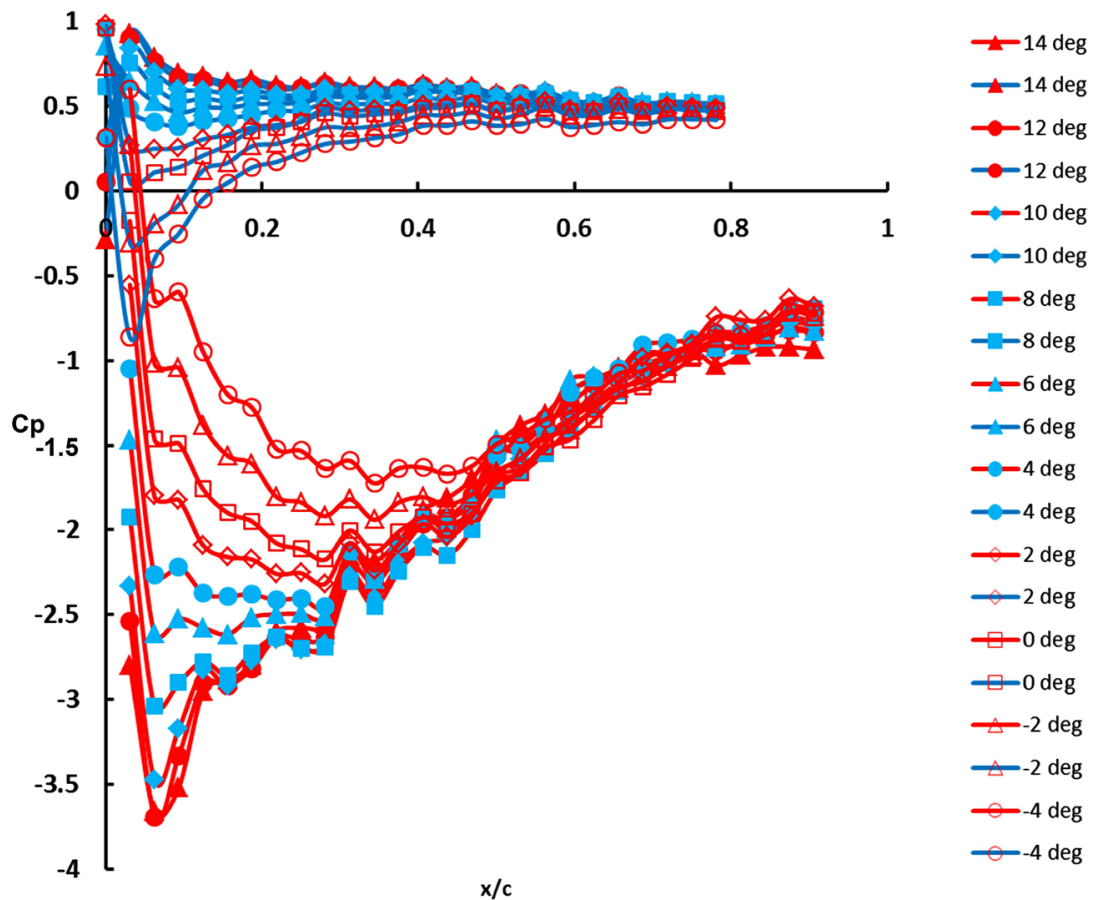

(b)

Figure 9. Pressure distribution with GF and VG. (a) Pressure distribution with $2 \%$ GF and VG; (b) Pressure distribution with 3\% GF and VG.

\subsection{Comparison of Variation of Coefficient of Pressure (Cp) with and without VG}

When we compare the pressure distribution of airfoil at higher angles of attack with and without GF, it is observed that there is a decrease in pressure at the 

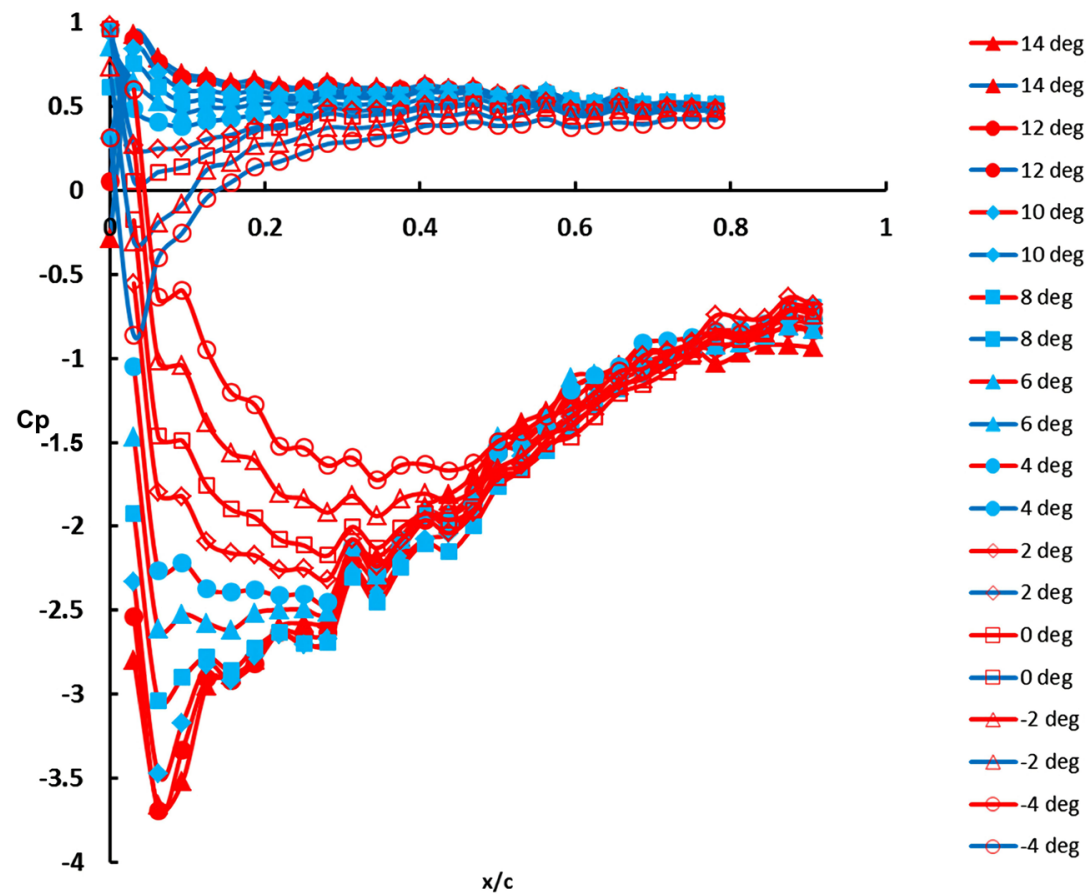

(a)
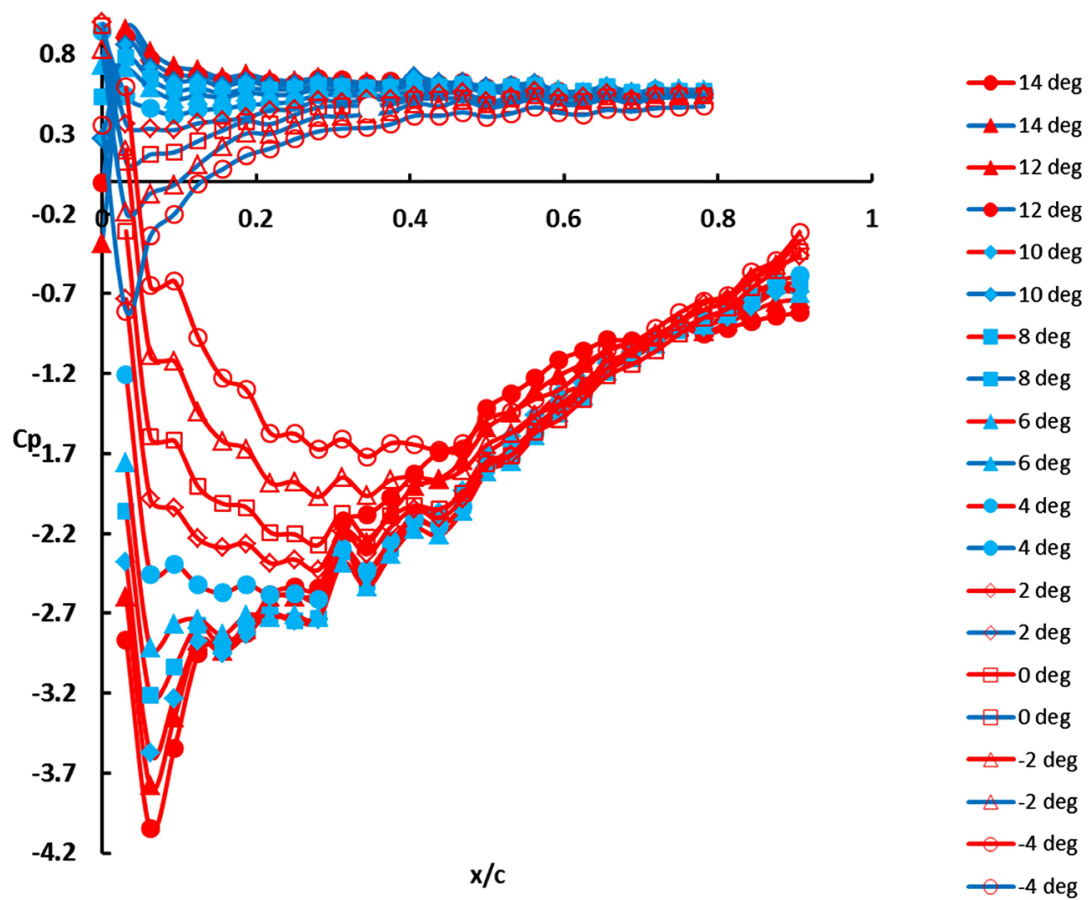

(b)

Figure 10. Pressure distribution with GF and VG. (a) Pressure Distribution with 4\% GF and VG; (b) Pressure distribution with 5\% GF and VG.

suction surface near the leading edge. It is because VG's are installed at the leading edge of the suction surface that trips the flow. The vital thing to notice is that on the suction surface of airfoil without GF, there is a flow separation starting close to $40 \%$ at $\mathrm{AoA}=14$ degree, $50 \%$ at $\mathrm{AoA}=12$ degree and $60 \%$ at $\mathrm{AoA}=$ 
10 degree. Whereas the airfoil with VG does not show this trend, which means flow does not separate easily as counter-rotating vortices energize the flow and allows it to remain attached to the suction surface at higher angles of attack as indicated in Figure 11 and Figure 12.

\subsection{Comparison of Variation of Coefficient of Pressure (Cp) with GF and VG}

When we compare pressure distribution on an airfoil with VG and GF at higher angles of attack, the pressure distribution on the pressure surface is modified, but there is a net reduction in suction peak. The overall effect is the decrease in lift, which indicates that the overall impact of both VG and GF is to increase the stall angle even though it reduces lift as shown in Figure 13 \& Figure 14.

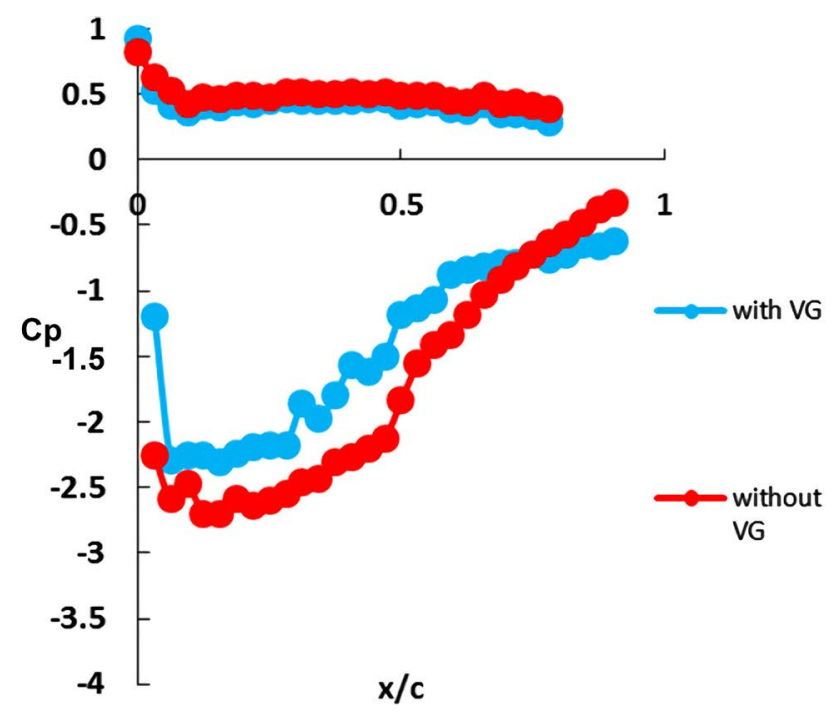

(a)

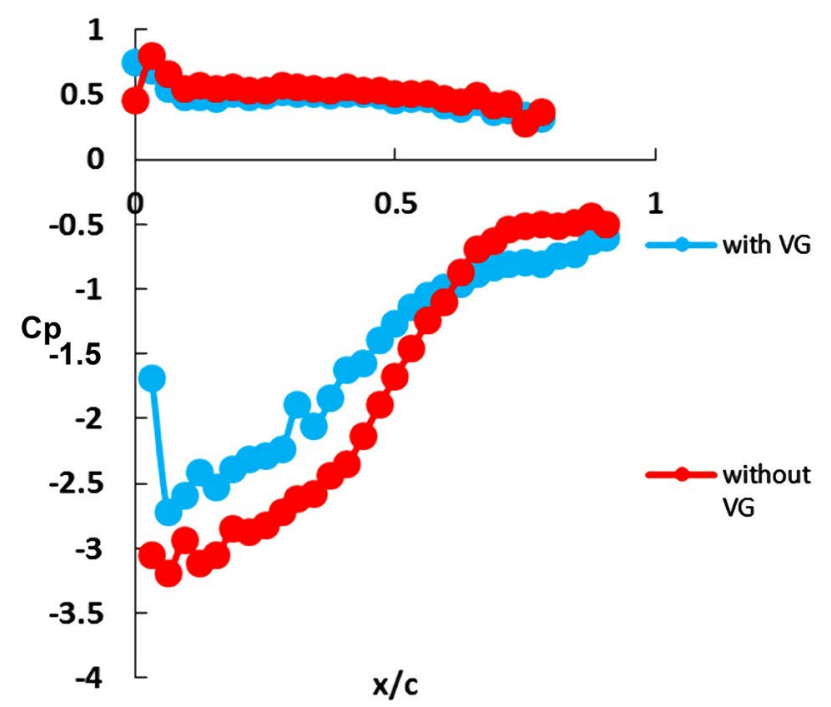

(b)

Figure 11. Variation of $\mathrm{Cp}$ with and without VG. (a) Pressure distribution at $\alpha=8$ degree with \& without VG; (b) Pressure distribution at $\alpha=10$ degree with \& without VG. 


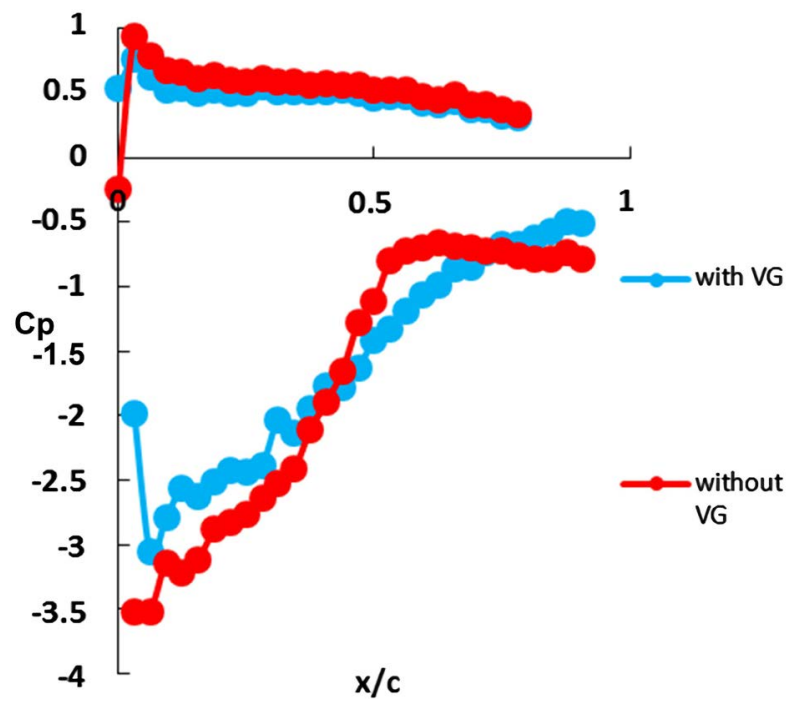

(a)

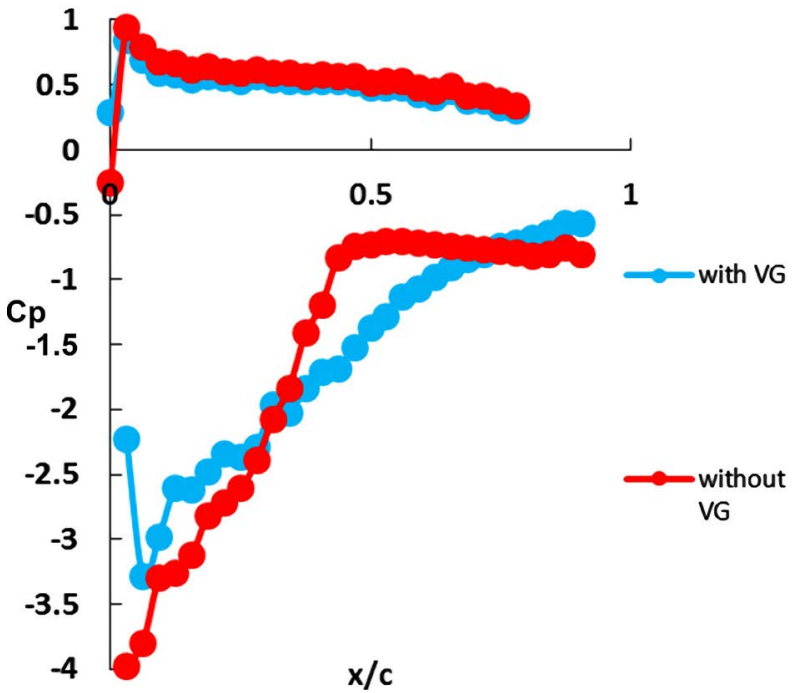

(b)

Figure 12. Variation of $\mathrm{Cp}$ with and without VG. (a) Pressure distribution at $\alpha=12$ degree with \& without VG; (b) Pressure distribution at $\alpha=14$ degree with \& without VG.

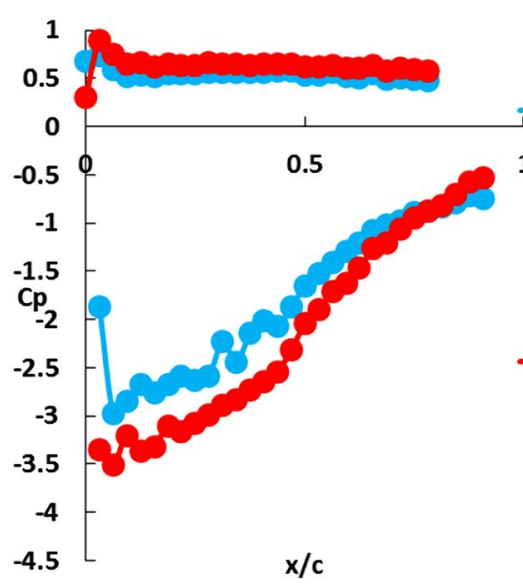

(a)

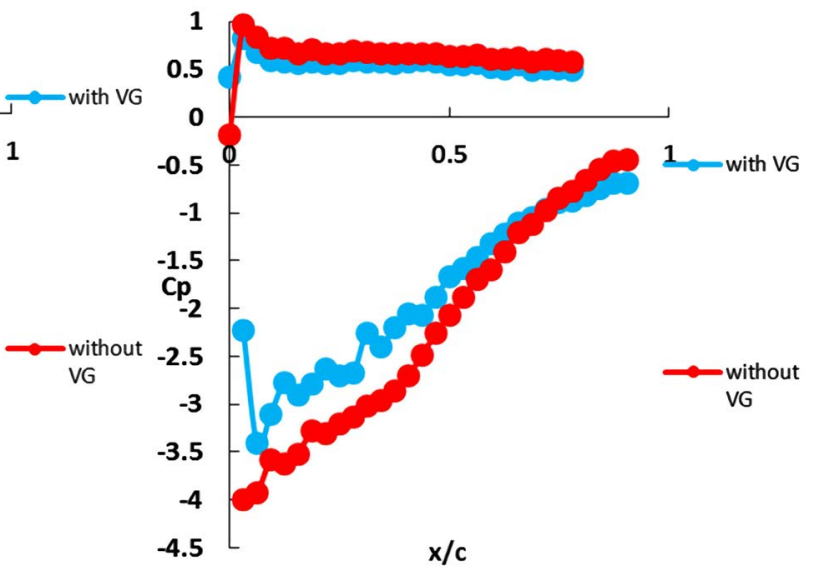

(b) 


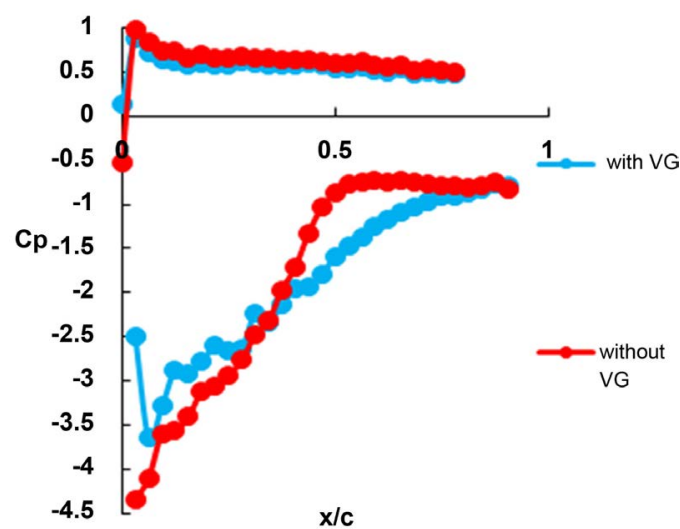

(c)

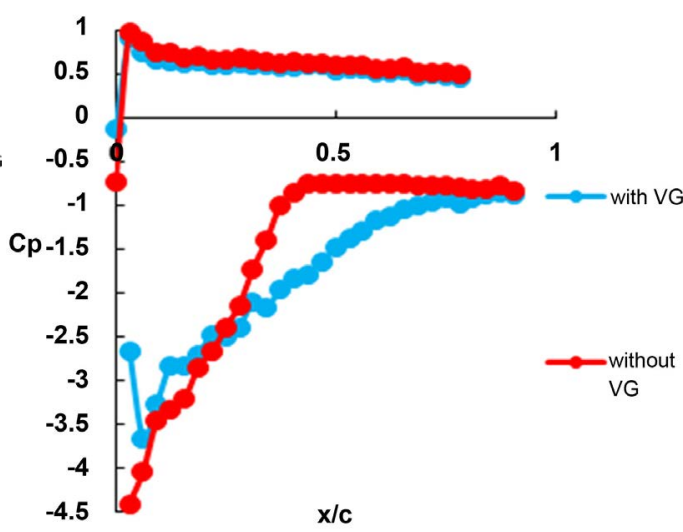

(d)

Figure 13. Variation of Cp with $2 \%$ GF and VG. (a) Pressure distribution at $\alpha=8$ degrees with $2 \%$ GF \& VG; (b) Pressure distribution at $\alpha=10$ degrees with 2\% GF \& VG; (c) Pressure distribution at $\alpha=12$ degrees with $2 \%$ GF \& VG; (d) Pressure distribution at $\alpha=14$ degrees with $2 \%$ GF \& VG.

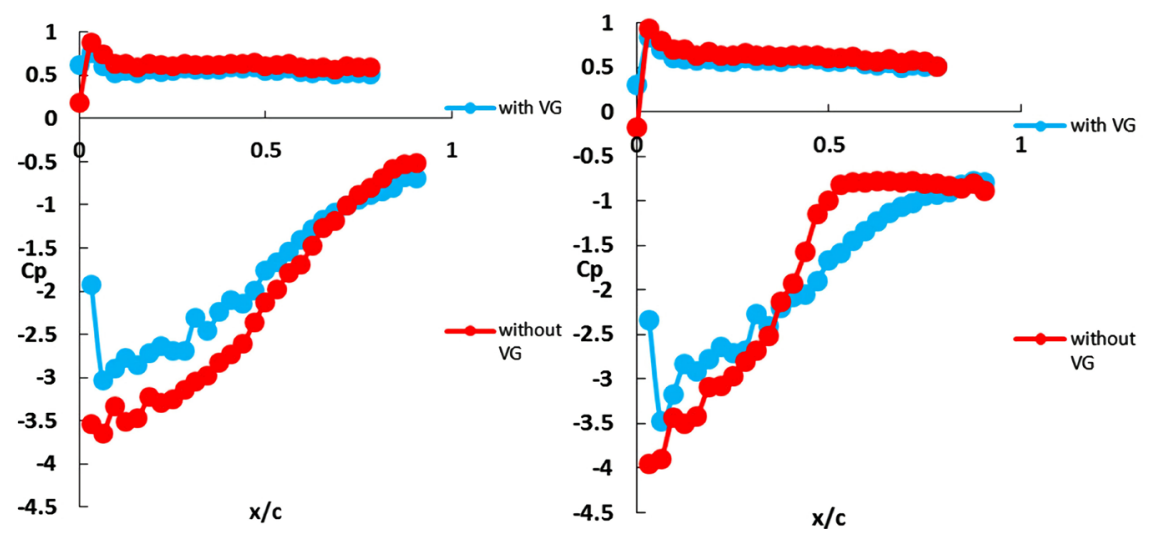

(a)

(b)

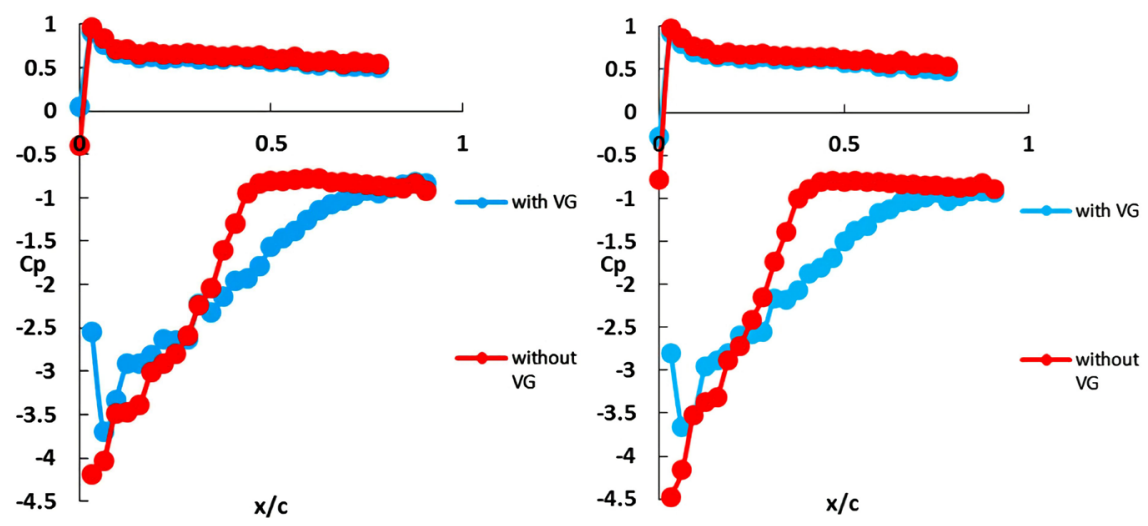

(c)

(d)

Figure 14. Variation of Cp with 3\% GF and VG. (a) Pressure distribution at $\alpha=8$ degrees with 3\% GF \& VG; (b) Pressure distribution at $\alpha=10$ degrees with 3\% GF \& VG; (c) Pressure distribution at $\alpha=12$ degrees with 3\% GF \& VG; (d) Pressure distribution at $\alpha=$ 14 degrees with $3 \%$ GF \& VG.

\subsection{Variation of Coefficient of Lift $\left(C_{l}\right)$ with and without GF}

Figure 15 shows the variation of $C_{l}$ with $\alpha$ for the clean airfoil and airfoil with 
various sizes of GF ( $2 \% \mathrm{C}$ to $5 \% \mathrm{C}$ ). It is observed that the addition of GF at the trailing edge increases the camber, which in turn increases the lift Coefficient. As GF height is increased progressively from $2 \% \mathrm{C}$ to $5 \% \mathrm{C}$ the stall angle reduces with little gain in lift. It was observed that Gurney Flap with $2 \% \mathrm{C}$ height performs better than other configurations which agrees well with existing literature that suggests optimum height for GF is around $2 \% \mathrm{C}$.

\subsection{Variation of Coefficient of Lift $\left(C_{l}\right)$ with GF and VG}

Figure 16 shows variation of $C_{l}$ with change in $\alpha$ for the airfoil with VG and GF, it was observed that addition of GF increases the camber and addition of VG at the leading edge reduces separation at the suction surface, and the combined effect of this configuration is to increase the lift coefficient and also increase the stall angle. But in comparison with GF only configuration (Figure 15) there is a reduction in value of $C_{l}$ but stall angle is enhanced with no sudden loss of lift. Thus, it can be said that addition of VG on airfoil with GF will reduce the lift but increase the stall angle as shown in Figure 16.

\subsection{Variation of Coefficient of Drag $\left(C_{d}\right)$ with and without GF}

The addition of GF theoretically should increase the drag, but it is quite evident from Figure 17 that airfoil with GF produces lesser drag in comparison to clean aerofoil. The reason for this is that the effective area of airfoil with GF reduces which leads to reduction in skin friction drag. Thus addition of GF not only increases the lift but also reduces the drag.

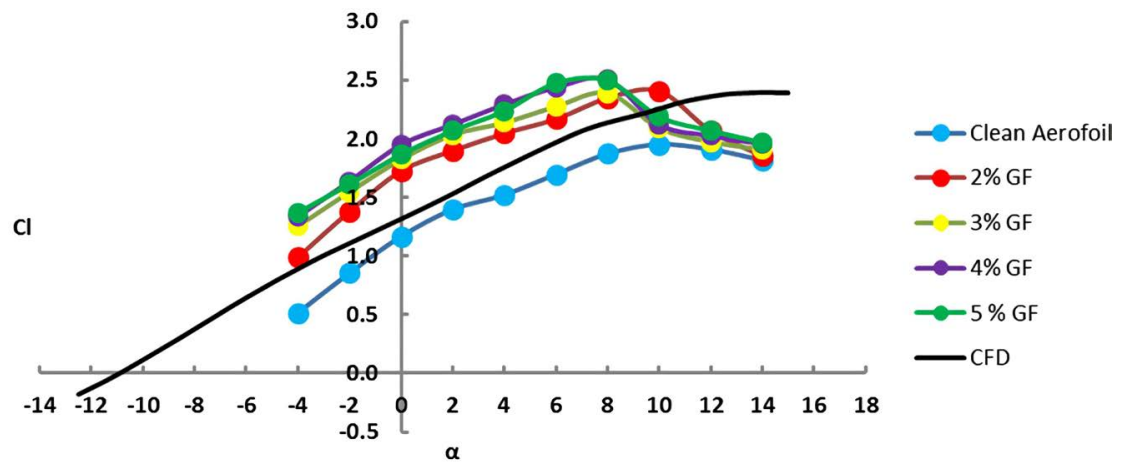

Figure 15. $C_{I}$ vs $\alpha$ with and without GF.

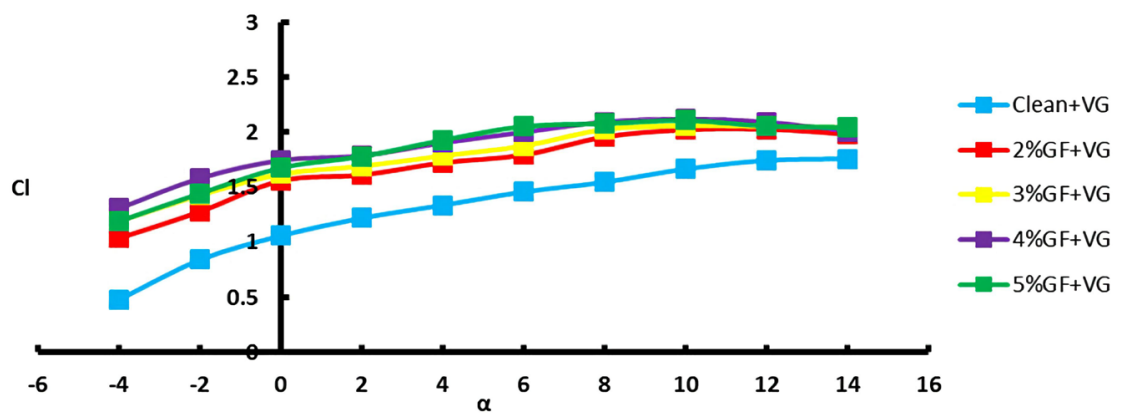

Figure 16. $C_{I}$ vs $\alpha$ with GF and VG. 


\subsection{Variation of Coefficient of Lift $\left(C_{d}\right)$ with GF and VG}

It can be seen from Figure 18 that drag has increased, which is due to the fact that VG will increase the skin friction drag which results in increase in total drag when compared with clean wing.

\subsection{Variation of $C_{l} / C_{d}$ with GF}

Figure 19 indicates that, with the addition of GF at the trailing edge the performance of airfoil increases in terms of $C_{l} / C_{d}$ when compared with clean airfoil. This is due to the fact that $C_{l}$ increases due to the increase in camber of the airfoil by addition of GF which contributes to enhanced aerodynamic efficiency. Also, it is observed that GF with $2 \%$ of the chord length performs best amongst other configurations.

\subsection{Variation of $C_{I} / C_{d}$ with GF and VG}

When both GF and VG were added on the Eppler 423 airfoil, the effect of VG is to increase the drag as it act as an obstruction in the flow at the leading edge thus causing reduction in $C_{l} / C_{d}$ as compared to an airfoil with only GF. Also it is observed that $3 \%$ GF with VG performs best in terms of increased $C_{l} / C_{d}$ when compared with other configurations (Figure 20).

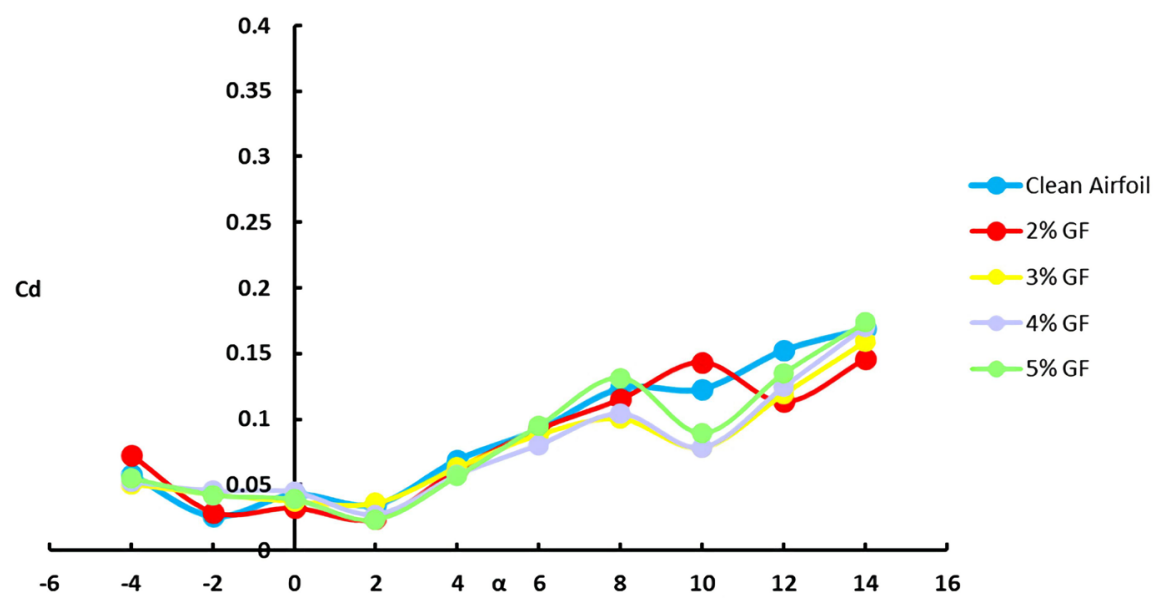

Figure 17. $C_{d}$ vs $\alpha$ with and without GF.

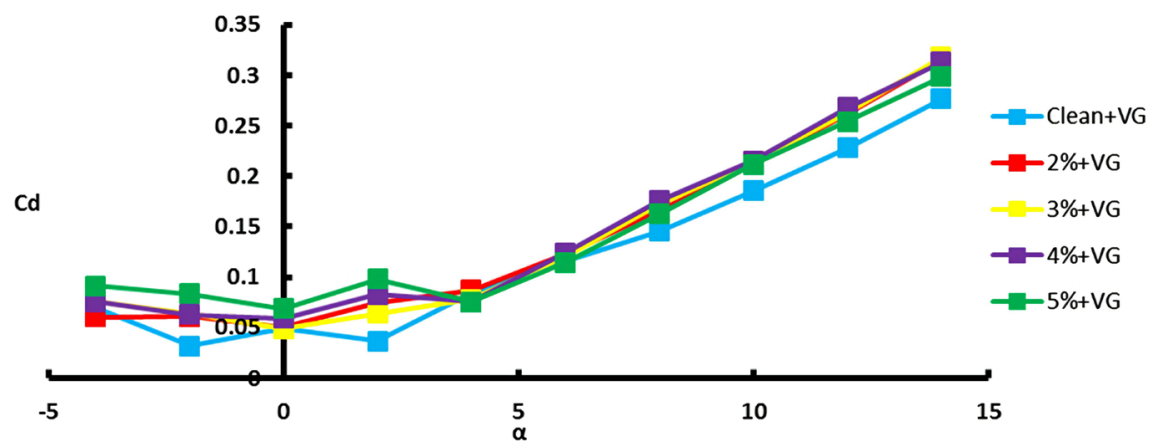

Figure 18. $C_{d}$ vs $\alpha$ with GF and VG. 

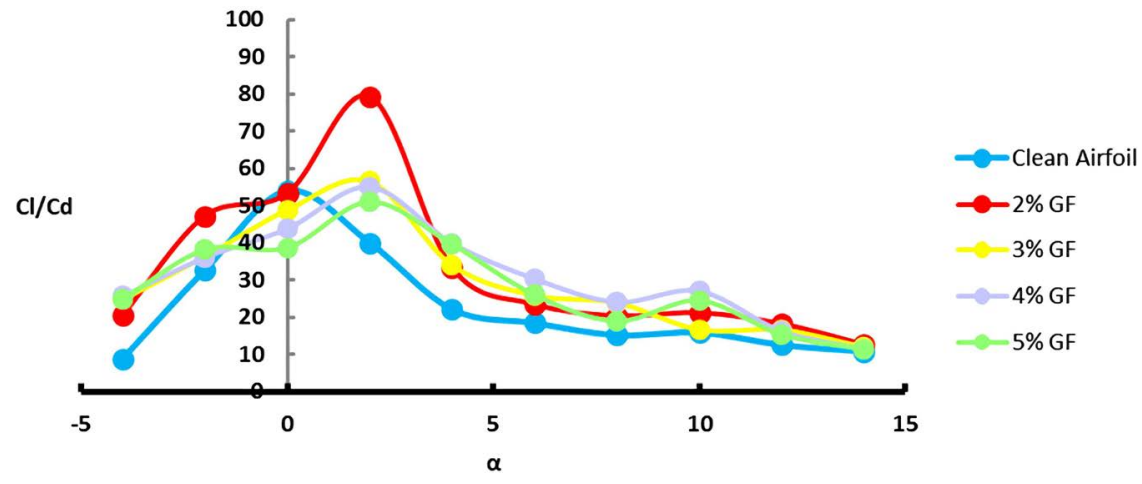

Figure 19. $C_{l} / C_{d}$ vs $\alpha$ with GF.

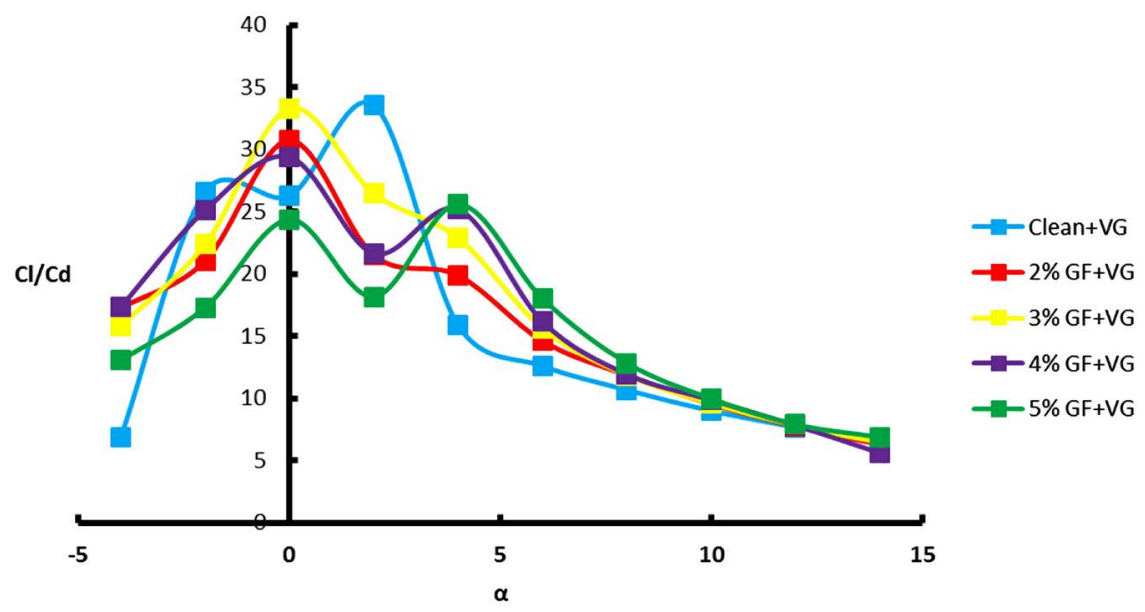

Figure 20. $C_{l} / C_{d}$ vs $\alpha$ with GF and VG.

\section{Conclusion}

At present many active and passive methods for reducing or for delaying the flow separation are being used to improve the aerodynamic performance of an airfoil. The net effect of using the GF is to increase the lift and decrease the stall angle. However, as we compare the net impact of adding a GF on a symmetric airfoil is more in comparison to cambered airfoil. This is due to the fact that flow gets reattached on the pressure surface leading to modified pressure pattern. Thus if the separation on the suction surface is reduced, the airfoil can behave as a high-speed airfoil. In the present study, experiments were conducted on Eppler 423 airfoil, which is highly cambered aerofoil. Results as obtained indicate that addition of GF will add camber to the airfoil, but amongst various sizes tested in this study the optimum GF size is $2 \%$ of the chord. The results after addition of VG show the modified pressure distribution on the suction surface, leading to an increase in stall angle, but there is a noticeable reduction in lift. VG at the leading edge, creates counter-rotating vortices that strengthen the flow thus reducing the flow separation but at the same time it also acts as a barrier at the leading edge thus adding as a source of drag. The position of VG was at the leading edge that needs to be optimized and should be $30 \%-40 \%$ of the chord as results without VG show the separation starts at around $40 \%$ of the chord at higher an- 
gles of attack. Also it was observed that combination of GF and VG underperforms when compared with GF only configuration this is due to the fact that addition of VG increases the drag thus reduction in $C_{l} / C_{d}$ but the advantage of adding VG at leading edge is that flow remains attached to the surface and thus stall angle is increased. Among all configurations Eppler 423 airfoil with GF having $2 \% \mathrm{C}$ height performs best due to reduced drag and higher lift generated due to the increase in camber. Also addition of VG on Eppler 423 airfoil with GF increases the stall angle and provides consistent lift even up till $\alpha=14$ degrees, which indicates that this combination can be used in applications that require sustained lift even at higher AoA.

\section{Conflicts of Interest}

The authors declare no conflicts of interest regarding the publication of this paper.

\section{References}

[1] https://en.wikipedia.org/wiki/Gurneyflap

[2] Liebeck, R.H. (1978) Design of Subsonic Airfoils for High Lift. Journal of Aircraft, 15, 547-561. https://doi.org/10.2514/3.58406

[3] Giguere, P., Dumas, G. and Lemay, J. (1997) Gurney Flap Scaling for an Optimum Lift to Drag Ratio. AIAA, 35, 1888-1890. https://doi.org/10.2514/2.49

[4] Li, Y.C., Wang, J.J., Tan, G.K. and Zhang, P.F. (2002) Effects of Gurney Flaps on the Lift Enhancement of a Cropped Nonslender Delta Wing. Experiment in Fluids, 32, 99-105. https://doi.org/10.1007/s003480200010

[5] Li, Y.C., Wang, J.J. and Zhang, P.F. (2003) Influences of Mounting Angles and Locations on the Effects of Gurney Flaps. Journal of Aircraft, 40, 494-498.

https://doi.org/10.2514/2.3144

[6] Neuhart, D.H. and Pendergraft, O.C. (1988) A Water Tunnel Study of Gurney Flaps. NASA TM-4071.

[7] Van Dam, C.P., Yen, D.T. and Vijgen, P.M.H.W. (1999) Gurney Flap Experiments of Airfoil and Wings. Journal of Aircraft, 36, 484-486.

https://doi.org/10.2514/2.2461

[8] Graham, M., Muradian, A. and Traub, L.W. (2018) Experimental Study on the Effect of Gurney Flap Thickness on Airfoil Performance. Journal of Aircraft, 35, 897-902. https://doi.org/10.2514/1.C034547

[9] Myose, R., Heron, I. and Papadakis, M. (1996) Effect of Gurney Flaps on a NACA 0011 Airfoil. AIAA Meeting Papers on Disc. https://doi.org/10.4271/961316

[10] Li, Y.C., Wang, J.J. and Zhang, P.F. (2002) Effect of Gurney Flaps on a NACA 0011 Airfoil. Flow, Turbulence, and Combustion.

[11] Giguère, P., Lemay, J. and Dumas, G. (1995) Gurney Flap Effects and Scaling for Low-Sped Aerofoils. AIAA, San Diego, 19-22 June 1995, 996-976.

https://doi.org/10.2514/6.1995-1881 


\section{List of Symbols}

AoA or $\alpha$ : Angle of Attack

H: Distance between Aerofoil and Ground

h/c: Height of Gurney Flap (\% C)

h: Height of Gurney Flap

C: Chord

$C_{\dot{d}}$ Coefficient of Drag

$C_{\dot{r}}$ Coefficient of Lift

$\mathrm{d} C / \mathrm{d} \alpha$ : Slope of Lift Curve

L/D: Lift to Drag Ratio

GF: Gurney Flap

VG: Vortex Generators

LE: Leading Edge

TE: Trailing Edge

BL: Boundary Layer 